\title{
Aquatic Training and Detraining on Fitness and Quality of Life in Fibromyalgia
}

PABLO TOMAS-CARUS ${ }^{1,2}$, ARJA HÄKKINEN $^{3}$, NARCIS GUSI $^{1}$, ALEJO LEAL $^{4}$, KEIJO HÄKKINEN $^{5}$, and ALFREDO ORTEGA-ALONSO

${ }^{1}$ Fitness and Lifequality Laboratory, Faculty of Sports Sciences, University of Extremadura, Cáceres, SPAIN; ${ }^{2}$ Department of Sports Sciences, Physical Activity and Health, University of Evora, Evora, PORTUGAL; ${ }^{3}$ Department of Physical Medicine and Rehabilitation, Jyväskylä Central Hospital, Jyväskylä, FINLAND; ${ }^{4}$ Hospital of Cáceres, Cáceres, SPAIN; ${ }^{5}$ Department of Biology of Physical Activity, University of Jyväskylä, Jyväskylä, FINLAND; and Department of Health Sciences, University of Jyväskylä, Jyväskylä, FINLAND

ABSTRACT

TOMAS-CARUS, P., A. HÄKKINEN, N. GUSI, A. LEAL, K. HÄKKINEN, and A. ORTEGA-ALONSO. Aquatic Training and Detraining on Fitness and Quality of Life in Fibromyalgia. Med. Sci. Sports Exerc., Vol. 39, No. 7, pp. 1044-1050, 2007. Purpose: To evaluate the effects of a 12 -wk period of aquatic training and subsequent detraining on health-related quality of life (HRQOL) and physical fitness in females with fibromyalgia. Methods: Thirty-four females with fibromyalgia were randomly assigned into two groups: an exercise group, who exercised for $60 \mathrm{~min}$ in warm water, three times a week $(N=17)$; and a control group, who continued their habitual leisure-time activities $(N=17)$. HRQOL was assessed using the Short Form 36 questionnaire and the Fibromyalgia Impact Questionnaire. Physical fitness was measured using the following tests: Canadian Aerobic Fitness, hand grip dynamometry, 10-m walking, 10-step stair climbing, and blind one-leg stance. Outcomes were measured at baseline, after treatment, and after 3 months of detraining. Results: After 12 wk of aquatic exercise, significant positive effects of aquatic training were found in physical function, body pain, general health perception, vitality, social function, role emotional problems and mental health, balance, and stair climbing. After the detraining period, only the improvements in body pain and role emotional problems were maintained. Conclusion: The present water exercise protocol improved some components of HRQOL, balance, and stair climbing in females with fibromyalgia, but regular exercise and higher intensities may be required to preserve most of these gains. Key Words: EXERCISE, POOL, HEALTH, PAIN, BALANCE, WOMEN

$\mathrm{P}$

atients with fibromyalgia (FM) present with muscle ain, weakness, and fatigue $(22,34)$ that may eventually lead to reduced physical activity and decreased quality of life (7). Some of the experimental physical therapies for FM have addressed the effects of exercising in warm water, trying to combine the revitalizing and strengthening effects of the physical exercise with the muscle-relaxation properties of bathing in warm water. These therapies have been shown to be effective for reducing symptoms and improving health-related quality of life (HRQOL), which normally has been evaluated with the Fibromyalgia Impact Questionnaire (FIQ) or the Short Form 36 (SF-36) $(3,8,16,20)$.

Most of these previous studies describe the effects of warm-water exercise on aerobic capacity and muscle

Address for correspondence: Narcís Gusi, Ph.D., Faculty of Sports Sciences, University of Extremadura Avenue, Universidad s/n, 10071 Cáceres, Spain; E-mail: ngusi@unex.es.

Submitted for publication July 2006

Accepted for publication March 2007

0195-9131/07/3907-1044/0

MEDICINE \& SCIENCE IN SPORTS \& EXERCISE

Copyright (C) 2007 by the American College of Sports Medicine

DOI: 10.1249/01.mss.0b0138059aec4 strength $(16,20)$, although to the best of our knowledge, data are scant or even missing regarding the effects of the warm-water exercise on other major abilities, such as walking speed, balance, or flexibility in patients with FM. Few of the previous trials have also evaluated a follow-up period in women with FM. All of these studies offered treatment or encouraged patients to continue exercising regularly during the follow-up period $(8,12,16,19)$. However, to know more precisely how long the adaptations caused by the therapy last, and to know of any possible need for continuous treatment, it is necessary to address the detraining effects after a certain period of physical treatment. This relevant topic has been analyzed by the present research group in the framework of a multidisciplinary controlled trial (ISRCTN83162243). A first study analyzing the effects of aquatic exercise and its subsequent detraining on muscular strength (as measured by an isokinetic dynamometer) and the time tradeoff utilities of HRQOL has been recently published (14). The current article presents the second study, which focuses on functional and physical fitness and on the generic and FM-specific attributes of HRQOL as main outcomes. Thus, the outcomes of the current article are addressed in relation to more pragmatic clinical issues related to sports medicine and exercise science. 